\title{
Hall and Ion-slip effects on unsteady MHD Bingham fluid flow with suction
}

\author{
Md. Tusher Mollah ${ }^{1 *}$, Muhammad Minarul Islam¹, Md. Mahmud Alam² \\ ${ }^{1}$ Department of Mathematics, Bangabandhu Sheikh Mujibur Rahman Science and Technology University, Gopalganj 8100, \\ Bangladesh \\ ${ }^{2}$ Mathematics Discipline, Khulna University, Khulna 9208, Bangladesh
}

Corresponding Author Email: tusher.bsmrstu@gmail.com

https://doi.org/10.18280/mmc_b.870402

Received: 15 May 2018

Accepted: 30 July 2018

\section{Keywords: \\ MHD, Bingham fluid, hall and Ion-slip current, suction, finite difference method}

\begin{abstract}
Hall and Ion-slip effects on unsteady MHD Bingham fluid flow through non-conducting parallel plates with uniform suction has been studied numerically. The fluid motion is subjected to uniform suction and external uniform magnetic field is applied perpendicular to the plates. The lower plate is stationary while upper plate moves with a constant velocity. Both plates are kept at different but constant temperatures. The governing non-linear coupled partial differential equations have been transformed into partial differential equations by usual transformations. The obtained equations have been solved numerically by the explicit finite difference method under the stability and convergence analysis. The effects of some important parameters on shear stress, Nusselt number as well as Primary Velocity, Secondary Velocity and Temperature distributions have been discussed graphically by MATLAB R2015a and Studio Developer FORTRAN 6.6a both. Finally, qualitative and quantitative comparisons of the present study with published results have been discussed.
\end{abstract}

\section{INTRODUCTION}

Bingham fluid is a special class of viscoelastic fluid that exhibit a linear behavior of shear stress versus shear rate once the fluid begins to flow. It behaves as a rigid body at low stress but flows as a viscous fluid at high stress. The MHD Bingham fluid flow is used in many geological and industry materials as a common mathematical model of mud flow in drilling engineering, and in the handling of slurries, lava, cement etc. A common example is toothpaste, which will not be extruded until a certain pressure is applied to the tube.

Bingham fluid is named after [1] who proposed its mathematical form in the article "An Investigation of the Laws of Plastic Flow". An exact description of friction loss for Bingham plastics in fully developed laminar pipe flow has been first considered by [2]. [3] described the rheology and flow of viscoelastic materials. The stability of Poiseuille flow of a Bingham fluid has been considered by [4]. The explicit calculation of the friction factor in pipeline flow of Bingham plastic fluids: a neural network approach has been investigated by [5]. [6] studied the effects of Hall current on unsteady MHD Couette flow and heat transfer on Bingham fluid with suction and injection. [7] investigated the numerical simulation of Taylor Couette flow of Bingham fluids. The explicit equations for laminar flow of Bingham plastic fluids has been considered by [8]. [9] studied the effect of Hall current on unsteady MHD free connective Couette flow of Bingham fluid with thermal radiation. The unsteady MHD Bingham fluid flow with Hall current and suction has been considered by [10]. [11] investigated the Hall effects on unsteady MHD flow of a NonNewtonian fluid through a Porous medium with uniform suction and injection. [12] studied the unsteady thermal boundary layer flows of a Bingham fluid in a porous medium.
[13] also considered the unsteady thermal boundary layer flows of a Bingham fluid in a porous medium following a sudden change in surface heat flux. [14] investigated the fluid flow through parallel plates in the presence of Hall current with inclined magnetic field in a rotating system. Recently the comparison of the obtained results and the published results has been reported by [15].

Hence our aim is to study the Hall and Ion-slip effects on unsteady MHD Bingham fluid flow through parallel plate with uniform suction. The explicit finite difference technique has been used to solve the dimensionless non-linear partial differential equations. The obtained results have been discussed graphically.

\section{MATHEMATICAL FORMULATION}

The laminar, incompressible Bingham fluid is assumed to be flowing between two infinite horizontal non-conducting parallel plates, which are located at $y= \pm h$ planes and extend from $x=0$ to $\infty$ and from $\mathrm{z}=0$ to $\infty$. The fluid motion is subjected to a uniform suction. The upper plate moves with a uniform velocity $U_{0}$ and the lower plate is stationary. Both the lower and upper plates are taken at two constant temperatures $T_{1}$ and $T_{2}$ respectively, where $T_{2}>T_{1}$. In the X-direction, a constant pressure gradient $\frac{d p}{d x}$ is applied along the fluid flow also a uniform magnetic field $B_{0}$ is acted perpendicular to the $\mathrm{X}$-direction which is undisturbed as the induced magnetic field is neglected by assuming a very small magnetic Reynolds number. Due to consideration of Hall effect, a Z-component for the velocity is expected to arise. For the uniform suction, $\frac{\partial u}{\partial x}=0$ and the continuity equation $\frac{\partial u}{\partial x}+\frac{\partial v}{\partial y}=0$ gives that 
$\frac{\partial v}{\partial y}=0$ thus the $\mathrm{Y}$-component of the velocity is constant. Hence the fluid velocity vector is given as $q=u(y, t) i+$ $v_{0} j+w(y, t) k$.

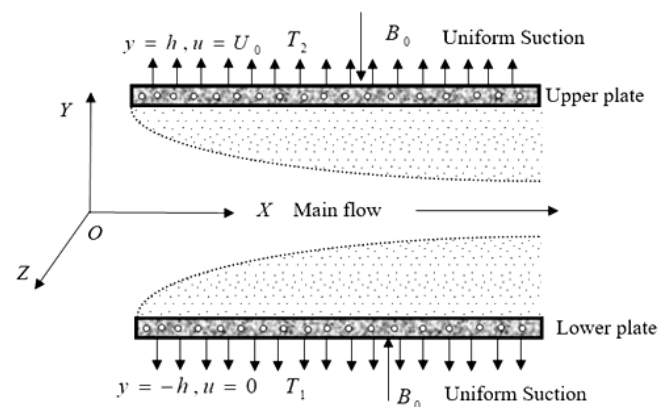

Figure 1. The physical configuration of the problem

Within the framework of the above assumptions, the equations related to the unsteady two-dimensional problem governed by the following system of coupled non-linear partial differential equations under the boundary-layer approximations, are given as follows:

Continuity equation:

$\frac{\partial v}{\partial y}=0$

Momentum equations:

$\frac{\partial u}{\partial t}-v_{0} \frac{\partial u}{\partial y}=-\frac{1}{\rho} \frac{d p}{d x}+\frac{1}{\rho} \frac{\partial}{\partial y}\left(\mu \frac{\partial u}{\partial y}\right)-\frac{1}{\rho} \frac{\sigma_{\mathrm{e}} B_{0}^{2}}{\alpha_{\mathrm{e}}^{2}+\beta_{\mathrm{e}}^{2}}\left(\alpha_{e} u+\beta_{e} w\right)$

$\frac{\partial w}{\partial t}-v_{0} \frac{\partial w}{\partial y}=\frac{1}{\rho} \frac{\partial}{\partial y}\left(\mu \frac{\partial w}{\partial y}\right)-\frac{1}{\rho} \frac{\sigma_{\mathrm{e}} B_{0}^{2}}{\alpha_{\mathrm{e}}^{2}+\beta_{\mathrm{e}}^{2}}\left(\alpha_{e} w-\beta_{e} u\right)$

Energy equation:

$$
\frac{\partial T}{\partial t}-v_{0} \frac{\partial T}{\partial y}=\frac{\kappa}{c_{p} \rho}\left(\frac{\partial^{2} T}{\partial y^{2}}\right)+\frac{\mu}{\rho c_{p}}\left[\left(\frac{\partial u}{\partial y}\right)^{2}+\left(\frac{\partial w}{\partial y}\right)^{2}\right]+\frac{1}{\rho c_{p}} \frac{\sigma_{\mathrm{e}}^{2} B_{0}^{2}}{\alpha_{\mathrm{e}}^{2}+\beta_{\mathrm{e}}^{2}}\left(u^{2}+w^{2}\right)
$$

$\mu=K+\frac{\tau_{0}}{\sqrt{\left(\frac{\partial u}{\partial y}\right)^{2}+\left(\frac{\partial w}{\partial y}\right)^{2}}}$

where and the corresponding initial and boundary conditions for the problem are as follows:

$t \leq 0, u=0, w=0, T=T_{1}$ everywhere

$$
\begin{aligned}
u & =0, w=0, T=T_{1} \quad \text { at } x=0 \\
t>0, & u=0, w=0, T=T_{1} \text { at } y=-h \\
u & =U_{0}, w=0, T=T_{2} \text { at } y=h
\end{aligned}
$$

where $\beta_{e}$ is the Hall parameter, $\beta_{i}$ is the ion-slip parameter, $\alpha_{e}=1+\beta_{i} \beta_{e}$.

Since the solution of the governing equation (1) - (5) under the initial conditions (6) and boundary conditions (7) will be based on the finite difference method for numerical solution, it is required to make the equations into dimensionless equations. The dimensionless quantities are given as follows:
$X=\frac{x}{h}, Y=\frac{y}{h}, U=\frac{u}{U_{0}}, W=\frac{w}{U_{0}}, P=\frac{p}{\rho U_{0}^{2}}, \tau=\frac{t U_{0}}{h}, \theta=\frac{T-T_{1}}{T_{2}-T_{1}}, \bar{\mu}=\frac{\mu}{K}$

The obtained dimensionless differential equations are given as follows:

$$
\begin{aligned}
& \frac{\partial U}{\partial \tau}-\frac{S}{R_{e}} \frac{\partial U}{\partial Y}=-\frac{d P}{d X}+\frac{1}{R_{e}}\left[\frac{\partial}{\partial Y}\left(\bar{\mu} \frac{\partial U}{\partial Y}\right)-\frac{H_{a}^{2}}{\alpha_{\mathrm{e}}^{2}+\beta_{\mathrm{e}}^{2}}\left(\alpha_{e} U+\beta_{e} W\right)\right] \\
& \frac{\partial W}{\partial \tau}-\frac{S}{R_{e}} \frac{\partial W}{\partial Y}=\frac{1}{R_{e}}\left[\frac{\partial}{\partial Y}\left(\bar{\mu} \frac{\partial W}{\partial Y}\right)-\frac{H_{a}^{2}}{\alpha_{\mathrm{e}}^{2}+\beta_{\mathrm{e}}^{2}}\left(\alpha_{e} W-\beta_{e} U\right)\right] \\
& \frac{\partial \theta}{\partial \tau}-\frac{S}{R_{e}} \frac{\partial \theta}{\partial Y}=\frac{1}{P_{r}} \frac{\partial^{2} \theta}{\partial Y^{2}}+E_{c} \bar{\mu}\left[\left(\frac{\partial U}{\partial Y}\right)^{2}+\left(\frac{\partial W}{\partial Y}\right)^{2}\right]+\frac{E_{c} H_{a}^{2}}{\alpha_{\mathrm{e}}^{2}+\beta_{\mathrm{e}}^{2}}\left(U^{2}+W^{2}\right) \\
& \bar{\mu}=1+\frac{\tau_{D}}{\sqrt{\left(\frac{\partial U}{\partial Y}\right)^{2}+\left(\frac{\partial W}{\partial Y}\right)^{2}}}
\end{aligned}
$$

And the dimensionless initial and boundary conditions can be written as follows:

$$
\begin{array}{cl}
\tau \leq 0, & U=0, W=0, \theta=0 \\
& U=0, W=0, \theta=0 \text { at } X=0 \\
\tau>0, & U=0, W=0, \theta=0 \text { at } Y=-1 \\
& U=1, W=0, \theta=1 \text { at } Y=1
\end{array}
$$

The non-dimensional parameters are given as follows:

$\tau_{D}=\frac{\tau_{0} h}{K U_{0}}$ (Bingham number or dimensionless yield stress); $R_{e}=\frac{\rho U_{0} h}{K}$ (Reynolds number); $P_{r}=\frac{\rho c_{p} U_{0} h}{k}$ (Prandtl number); $E_{C}=\frac{U_{0} K}{\rho c_{p} h\left(T_{2}-T_{1}\right)}$ (Eckert number); $H a^{2}=\frac{\sigma B_{0}^{2} h^{2}}{K}$ (Hartmann number squared); $S=\frac{\rho V_{0} h}{K}$ (Suction Parameter)

\section{SHEAR STRESS AND NUSSELT NUMBER}

The effects of various parameters on shear stress have been studied from the velocity profile. The shear stress in $\mathrm{X}$ direction for stationary wall is $\tau_{w 1} \equiv$ $\left[\bar{\mu} \sqrt{\left(\frac{\partial U}{\partial Y}\right)^{2}+\left(\frac{\partial W}{\partial Y}\right)^{2}}\right]_{Y=-1}$ and for moving wall is $\tau_{w 2} \equiv$ $\left[\bar{\mu} \sqrt{\left(\frac{\partial U}{\partial Y}\right)^{2}+\left(\frac{\partial W}{\partial Y}\right)^{2}}\right]_{Y=1}$.

Also, the effects of various parameters on Nusselt number have been studied from the temperature profile. Nusselt number in X-direction for stationary wall is $N_{u 1} \equiv \frac{\left(\frac{\partial T}{\partial Y}\right)_{Y=-1}}{-T_{m}}$ and for moving wall is $N_{u 2} \equiv \frac{\left(\frac{\partial T}{\partial Y}\right)_{Y=1}}{-\left(T_{m}-1\right)}$, where $T_{m}$ is the dimensionless mean fluid temperature and is given by $T_{m}=$ $\frac{\int_{-1}^{1} U \theta d Y}{\int_{-1}^{1} U d Y}$.

\section{CALCULATION TECHNIQUE}

To solve the dimensionless partial differential equations (9) 
- (12) by the explicit finite difference method subject to the boundary conditions, a set of finite difference equations is required. For which, the region within the boundary layer is divided into a grid or mesh of lines perpendicular to Y-axis where $\mathrm{Y}$-axis is normal to the plate as shown in Figure 2.

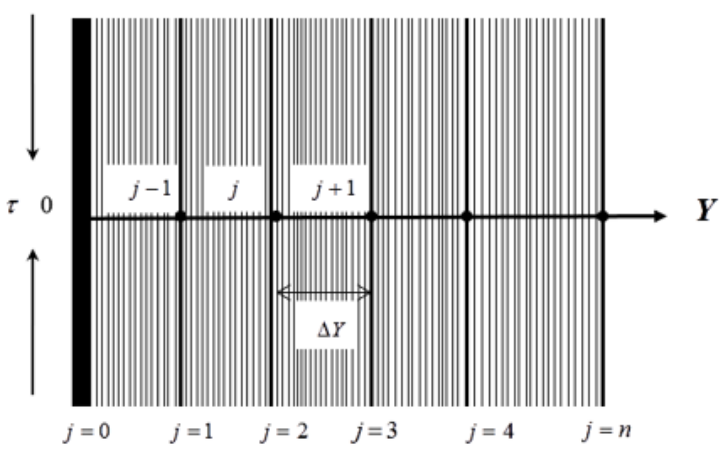

Figure 2. Finite difference space grid

Here it is assumed that the maximum length of boundary layer is $Y(=2)_{\max }$ as corresponding to $Y \rightarrow \infty$ i.e. $Y$ varies from 0 to 2 . The number of grids spacing $n=100$ along the $\mathrm{Y}$-directions. It is assumed that $\Delta Y$ is constant mesh size $\mathrm{Y}$ direction and taken as follows:

$\Delta Y=0.02(0 \leq x \leq 100)$; with the smaller time-step, $\Delta \tau=0.0001$

Let $U^{\prime}, W^{\prime}$ and $\theta^{\prime}$ denote the values of $U, W$ and $\theta$ at the end of a time-step respectively. Using the explicit finite difference approximation, the following appropriate set of finite difference equations are obtained as follows:

$$
\begin{aligned}
\frac{U_{i}^{\prime}-U_{i}}{\Delta \tau} & -\frac{S}{R_{e}} \frac{U_{i}-U_{i-1}}{\Delta Y}=-\frac{d P}{d X} \\
& +\frac{1}{R_{e}}\left[\left(\frac{\bar{\mu}_{i}-\bar{\mu}_{i-1}}{\Delta Y}\right)\left(\frac{U_{i}-U_{i-1}}{\Delta Y}\right)+\bar{\mu}_{i}\left(\frac{U_{i+1}-2 U_{i}+U_{i-1}}{(\Delta Y)^{2}}\right)-\frac{H_{a}^{2}}{\alpha_{\mathrm{e}}^{2}+\beta_{\mathrm{e}}^{2}}\left(\alpha_{e} U_{i}+\beta_{e} W_{i}\right)\right]
\end{aligned}
$$

$$
\begin{aligned}
& \frac{W_{i}^{\prime}-W_{i}}{\Delta \tau}-\frac{S}{R_{e}} \frac{W_{i}-W_{i-1}}{\Delta Y}= \\
& \quad+\frac{1}{R_{e}}\left[\left(\frac{\bar{\mu}_{i}-\bar{\mu}_{i-1}}{\Delta Y}\right)\left(\frac{W_{i}-W_{i-1}}{\Delta Y}\right)+\bar{\mu}_{i}\left(\frac{W_{i+1}-2 W_{i}+W_{i-1}}{(\Delta Y)^{2}}\right)-\frac{H_{a}^{2}}{\alpha_{\mathrm{e}}^{2}+\beta_{\mathrm{e}}^{2}}\left(\alpha_{e} W_{i}-\beta_{e} U_{i}\right)\right]
\end{aligned}
$$

$$
\begin{aligned}
& \frac{\theta_{i}^{\prime}-\theta_{i}}{\Delta \tau}-\frac{S}{R_{e}} \frac{\theta_{i}-\theta_{i-1}}{\Delta Y}=\frac{1}{P_{r}} \frac{\theta_{i+1}-2 \theta_{i}+\theta_{i-1}}{(\Delta Y)^{2}} \\
& \quad+E_{c}\left(\bar{\mu}_{i}\right)\left[\left(\frac{U_{i}-U_{i-1}}{\Delta Y}\right)^{2}+\left(\frac{W_{i}-W_{i-1}}{\Delta Y}\right)^{2}\right]+\frac{E_{c} H_{a}^{2}}{\alpha_{\mathrm{e}}^{2}+\beta_{\mathrm{e}}^{2}}\left(U_{i}^{2}+W_{i}^{2}\right)
\end{aligned}
$$

$$
\bar{\mu}_{i}=1+\frac{\tau_{D}}{\sqrt{\left(\frac{U_{i}-U_{i-1}}{\Delta Y}\right)^{2}+\left(\frac{W_{i}-W_{i-1}}{\Delta Y}\right)^{2}}}
$$

And the boundary conditions with the finite difference scheme are given as follows: $U_{L}=0, W_{L}=0, \theta_{L}=0$ at $L=$ $-1 U_{L}=1, W_{L}=0, \theta_{L}=1$ at $L=1$ Here the subscripts $i$ designate the grid points with ${ }^{y}$ coordinates.

\section{STABILITY AND CONVERGENCE ANALYSIS}

Since an explicit procedure is being used, the analysis will remain incomplete unless the stability and convergence of the finite difference scheme are discussed. For the constant mesh sizes the stability and convergence criteria finally can be written as follows:

$\frac{\Delta \tau}{2 R_{e}} \frac{H_{a}^{2}}{\alpha_{\mathrm{e}}^{2}+\beta_{\mathrm{e}}^{2}} \alpha_{e}-\frac{S \Delta \tau}{R_{e} \Delta Y} \leq 1$

$\frac{2 \Delta \tau}{P_{r}(\Delta Y)^{2}}-\frac{S \Delta \tau}{R_{e} \Delta Y} \leq 1$

Using $\Delta Y=0.02, \Delta \tau=0.0001$ and the initial conditions, the above equations (19) and (20) gives $R_{e} \geq 0.06, P_{r} \geq$ $-0.6, \beta_{i} \geq 1, H_{a} \leq 10$ and $S \leq-1$ with $\beta_{e}=0.10$,and $E_{c}=$ 0.01

\section{RESULTS AND DISCUSSION}

To investigate the physical conditions of the developed mathematical model it has been obtained the numerical values of the primary velocity $U$, secondary velocity $W$ and temperature $\theta$ within the boundary layer for the laminar boundary layer flow. The effect of Hall parameter $\beta_{e}$, Ion-slip parameter $\beta_{i}$, where $\alpha_{e}=1+\beta_{i} \beta_{e}$, Hartmann number $H_{a}$ and Suction parameter $S$ on velocity and temperature distributions as well as local shear stress and local Nusselt number at both stationary and moving plates are discussed. For brevity, the effect of other parameters such as Reynolds number $R_{e}$, Prandtl number $P_{r}$, Eckert number $E_{c}$ and Bingham number $\tau_{D}$ are not shown.

\subsection{Validation}

To verify the effects of grid space for $m$ and $n$, the computations have been carried out for three different grid spaces such as $n=40, n=80$ and $n=100$ are shown in Fig.3. But a negligible change has seen among these curves. According to this situation, the results of primary velocity, secondary velocity and temperature distributions have been carried out for $n=100$.

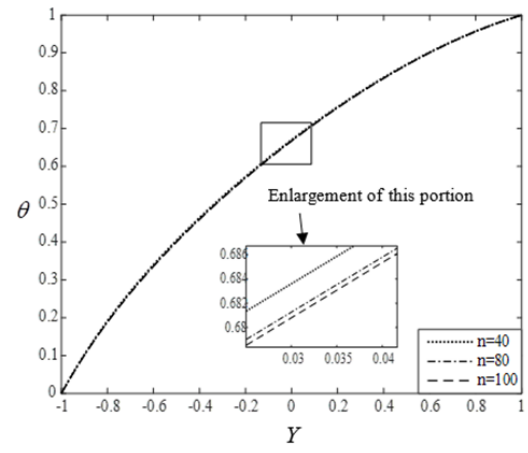

Figure 3. Justifying grid space; Where $\mathrm{S}=-1.00, \beta_{i}=$ $3.00, H_{a}=3.00, \beta_{e}=2.00, E_{c}=0.01, P_{r}=1.00$ and $\tau_{D}=$ 0.1 at time $\tau=4.00$ (Steady State)

The verification of the finite difference solutions by MATLAB R2015a is achieved with the finite difference solutions by Studio Developer FORTRAN (SDF) 6.6a. The same results are obtained from the above-mentioned tools. The computations for Primary velocity $U$ and local shear stress 
$\tau_{w 2}$ with respect to dimensionless time $\tau$ at moving plate have been shown in Fig.4 for different values of suction parameter.

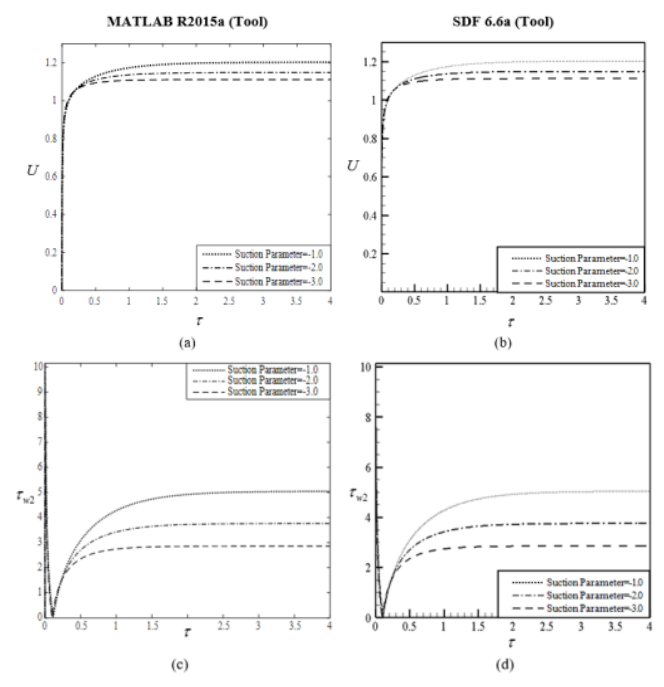

Figure 4. Effect of Suction parameter (S) on (a) Primary velocity at moving plate (MATLAB R2015a); (b) Primary velocity at moving plate (SDF 6.6a); (c) local shear stress at moving plate (MATLAB R2015a); (d) Local shear stress at moving plate (SDF 6.6a); where $\beta_{i}=3.00, H_{a}=3.00, \beta_{e}=$ $0.10, R_{e}=2.00, E_{c}=0.01 P_{\tau}=1.00$ and $\tau_{D}=0.1$

\subsection{Steady state solution}

In order to verify the effects of time step size $\Delta \tau$, the computations have been carried out for six different time step sizes such as $\tau=0.20,0.70,1.20,3.00,4.00$ and 5.00. It is observed that, the result of computations for $\mathrm{U}, W$ and $\theta$, however shows so little changes after $\tau=3.00$ also shows a negligible change after $\tau=4.00$. Thus the solutions of all variables for $\tau=4.00$ are essentially the steady-state solutions, which are shown in Fig.5. Hence the further computations have been carried out for $\tau=4.00$.

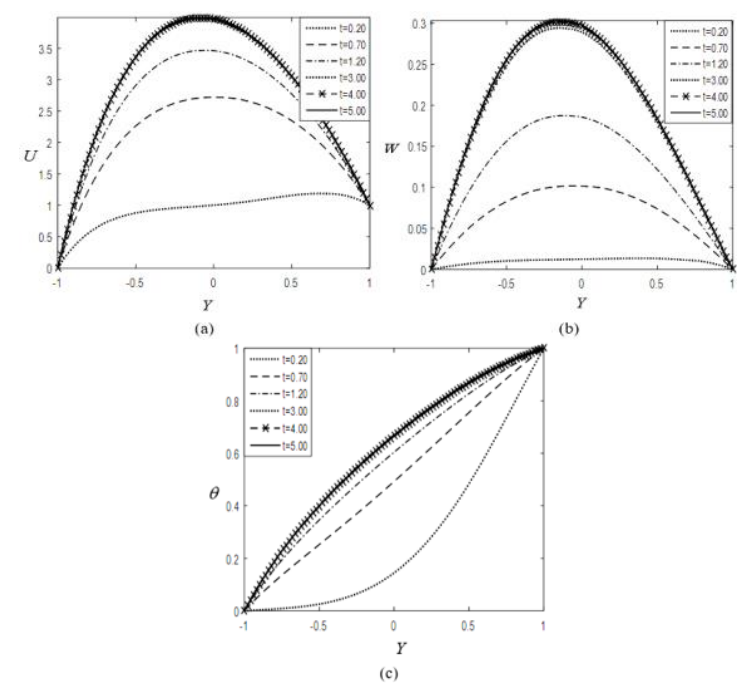

Figure 5. Illustration of time Variation for (a) Primary velocity, (b) Secondary velocity and (c) Temperature distributions, where $\mathrm{S}=-1.00, \beta_{i}=3.00, H_{a}=3.00, \beta_{e}=$ $0.10, R_{e}=2.00, E_{c}=0.01 P_{r}=1.00$ and $\tau_{D}=0.1$

It is seen from Fig.5 it is clear that the primary velocity, secondary velocity and temperature profiles reach their steady state monotonically. It also should be mentioned that temperature profiles reach the steady state faster than both the primary velocity and secondary velocity. Again the secondary velocity reaches steady state briskly in the comparison with primary velocity and temperature both.

\subsection{Effect of parameters}

In order to get the clear concept of physical properties of the problem, the effects of four parameters namely Hall parameter $\beta_{e}$, Ion-slip parameter $\beta_{i}$, Hartmann number $H_{a}$ and Suction parameter $S$ in the presence of Reynolds number $\left(R_{e}=3.00\right)$, Prandtl number $\left(P_{r}=1.00\right)$, Eckert number $\left(E_{c}=0.01\right)$ and Bingham number $\left(\tau_{D}=0.10\right)$ are represented graphically through Figs.6 - 17. For brevity, the effect of other parameters such as Reynolds number $R_{e}$, Prandtl number $P_{r}$, Eckert number $E_{c}$ and Bingham number $\tau_{D}$ are not shown.

The effects of Hartmann number $H_{a}$ on primary velocity, secondary velocity and temperature distributions as well as local shear stress, and local Nusselt number at both stationary and moving plates are presented in Figs.6 - 8. From Fig. $6(\mathrm{a}, \mathrm{b}, \mathrm{c}, \mathrm{d})$, it is observed that the primary velocity and the local shear stress decreases with the increase of $H_{a}$ at both stationary and moving plates. It is observed from Fig.7(a,b) that the secondary velocity increases with the increase of $H_{a}$ at both stationary and moving plates. From Fig.8(a,b,c,d), it is shown that the temperature decreases while the local Nusselt number increases with the increase of $H_{a}$ at stationary plate. Again, the temperature and the local Nusselt number decreases with the increase of $H_{a}$ at moving plate.

Again, the effects of Hall parameter $\beta_{e}$ on primary velocity, secondary velocity and temperature distributions as well as local shear stress, and local Nusselt number at both stationary and moving plates are presented in Figs.9 - 11. From Fig.9(a,b,c,d), it is observed that the primary velocity as well as the local shear stress increases with the increase of $\beta_{e}$ at both stationary and moving plates. It is shown from Fig. 10(a,b) that the secondary velocity decreases with the increase of $\beta_{e} \beta_{e}$ at both stationary and moving plates. From Fig.11(a,b,c,d), it is detected that the temperature increases with the increase of $\beta_{e}$ at both stationary and moving plates. Again, the local Nusselt number decreases with the increase of $\beta_{e}$ at both stationary and moving plates. This is due to the fact an increase in $\beta_{e}$ decreases effective conductivity $\left(\frac{\sigma_{e}^{2}}{\alpha_{e}^{2}+\beta_{e}}\right)$, hence magnetic dumping force on $U$.

Further the effects of Ion-slip parameter $\beta_{i}$ on primary velocity, secondary velocity and temperature distributions as well as local shear stress, and local Nusselt number at both stationary and moving plates are presented in Figs.12 - 14 . From Fig.12(a,b,c,d), it is observed that the primary velocity as well as the local shear stress increases with the increase of $\beta_{i}$ at both stationary and moving plates. It is shown from Fig.13(a,b) that the secondary velocity decreases with the increase of $\beta_{i}$ at both stationary and moving plates. It is observed from Fig.14(a,b,c,d) that the temperature increases with the increase of $\beta_{i}$ at both stationary and moving plates. Again, the local Nusselt number decreases with the increase of $\beta_{i}$ at both stationary and moving plates.

The effects of Suction parameter $S$ on primary velocity, secondary velocity and temperature distributions as well as local shear stress, and local Nusselt number at both stationary and moving plates are presented in Figs.15 - 17. From 
Fig.15(a,b,c,d), it is observed that the primary velocity and the local shear stress decreases with the increase of $S$ at stationary plate while at moving plate the primary velocity and the local shear stress increases with the increase of $S$. It is realized from Fig.16(a,b) that the secondary velocity decreases with the increase of $S$ at stationary plate while at moving plate it increases with the increase of $S$. It is observed from Fig.14(a,b,c,d) that the temperature decreases with the increase of $S$ at both stationary and moving plates. Again, the local Nusselt number increases with the increase of $S$ at both stationary and moving plates.
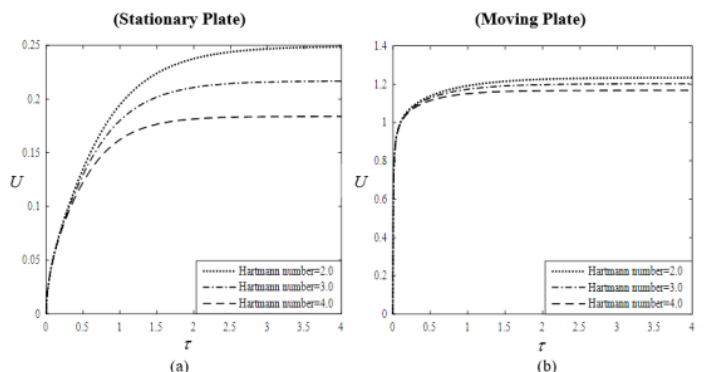

(b)
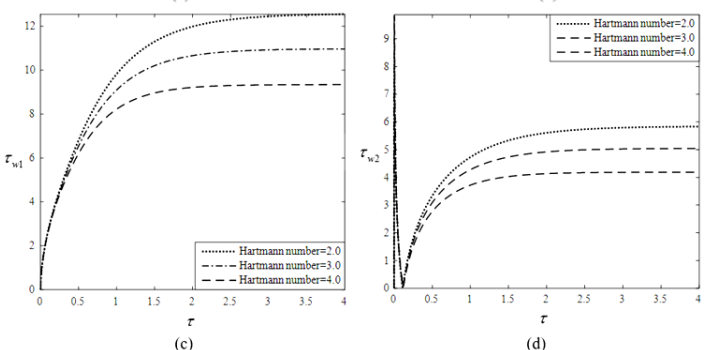

Figure 6. Effect of Hartmann number $\left(H_{a}\right)$ on (a) Primary velocity (stationary plate); (b) Primary velocity (moving plate); (c) local shear stress (stationary plate); (d) Local shear stress (moving plate); where $\mathrm{S}=-1.00, \beta_{i}=3.00, \beta_{e}=$

$$
0.10, R_{e}=2.00, E_{c}=0.01 P_{r}=1.00 \text { and } \tau_{D}=0.1
$$

Fig. 6 shows that the primary velocity as well as the local shear stress decreases with the increase of Hartmann number $H_{a}$ at both stationary and moving plates.

The primary velocity at moving plate is more than the primary velocity at stationary plate while the local shear stress at moving plate is fewer than the local shear stress at stationary plate.

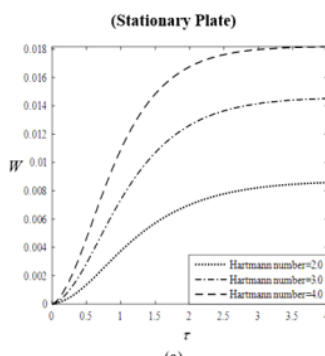

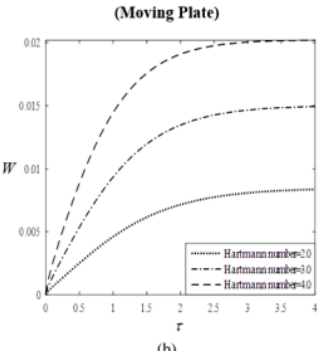

Figure 7. Effect of Hartmann number $\left(H_{a}\right)$ on (a) Secondary velocity (stationary plate); (b) Secondary velocity (moving plate); where $\mathrm{S}=-1.00, \beta_{i}=3.00, \beta_{e}=0.10, R_{e}=$

$$
2.00, E_{c}=0.01 P_{r}=1.00 \text { and } \tau_{D}=0.1
$$

Fig. 7 shows that the secondary velocity increases with the increase of Hartmann number $H_{a}$ at both stationary and moving plates.

The secondary velocity at moving plate is more than the secondary velocity at stationary plate.

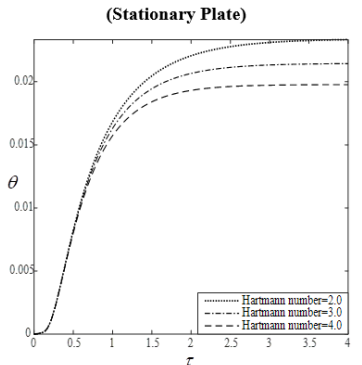

(a)

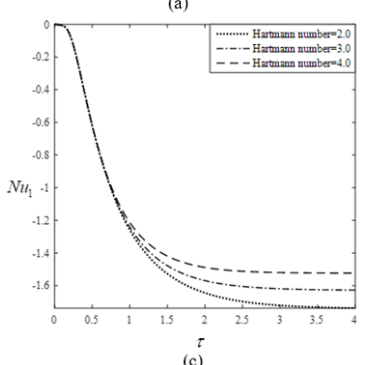

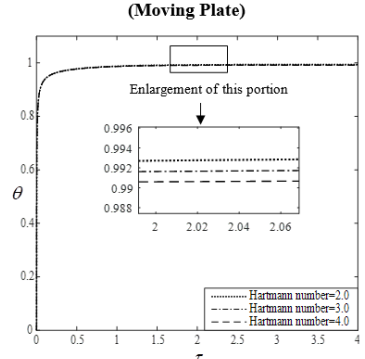

(b)

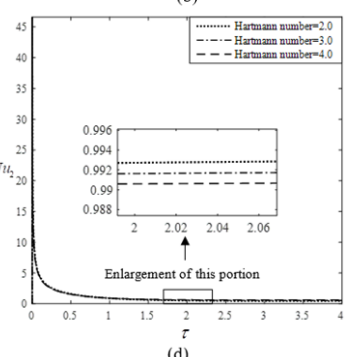

Figure 8. Effect of Hartmann number $\left(H_{a}\right)$ on (a) Temperature (stationary plate); (b) Temperature velocity (moving plate); (c) local Nusselt number (stationary plate);

(d) Local Nusselt number (moving plate); where $\mathrm{S}=$ $-1.00, \beta_{i}=3.00, \beta_{e}=0.10, R_{e}=2.00, E_{c}=0.01 P_{r}=$ 1.00 and $\tau_{D}=0.1$

Fig. 8 shows that the temperature decreases while the local Nusselt number increases with the increase of Hartmann number $H_{a}$ at stationary plate. Also, the temperature and the local Nusselt number decreases with the increase of Hartmann number $H_{a}$ at moving plate.

The temperature and local Nusselt number at moving plate is more than the temperature and local Nusselt number at stationary plate.
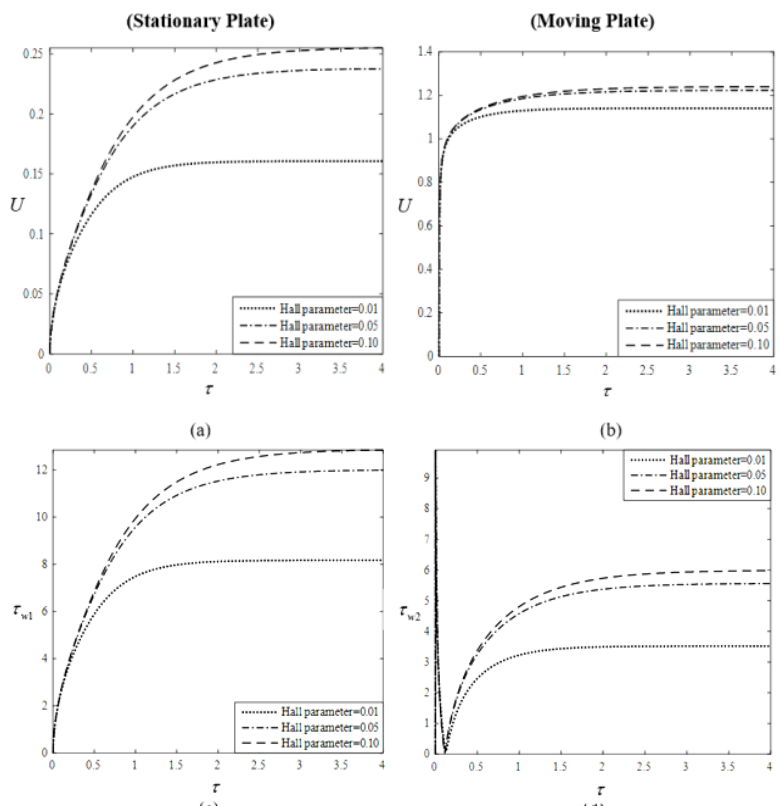

Figure 9. Effect of Hall parameter $\left(\beta_{e}\right)$ on (a) Primary Velocity (stationary plate); (b) Primary Velocity (moving plate); (c) local shear stress (stationary plate); (d) Local shear stress (moving plate); where $\mathrm{S}=-1.00, \beta_{i}=3.00, H_{a}=$ $3.00, R_{e}=2.00, E_{c}=0.01 P_{r}=1.00$ and $\tau_{D}=0.1$

Figure 9 shows that the primary velocity as well as the local 
shear stress increases with the increase of Hall parameter $\beta_{e}$ at both stationary and moving plates.

The primary velocity at moving plate is more than the primary velocity at stationary plate while the local shear stress at moving plate is fewer than the local shear stress at stationary plate.
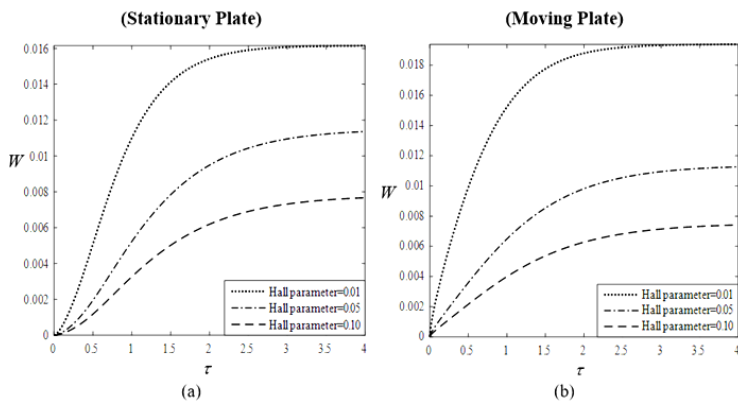

Figure 10. Effect of Hall parameter $\left(\beta_{e}\right)$ on (a) Secondary velocity (stationary plate); (b) Secondary velocity (moving plate); where $\mathrm{S}=-1.00, \beta_{i}=3.00, H_{a}=3.00, R_{e}=$

$$
2.00, E_{c}=0.01 P_{r}=1.00 \text { and } \tau_{D}=0.1
$$

Figure 10 shows that the secondary velocity decreases with the increase of Hall parameter $\beta_{e}$ at both stationary and moving plates.

The secondary velocity at moving plate is more than the secondary velocity at stationary plate.

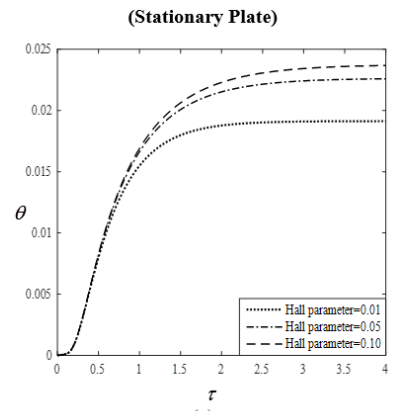

(a)

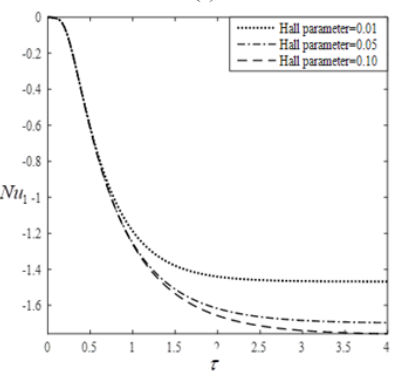

(c)

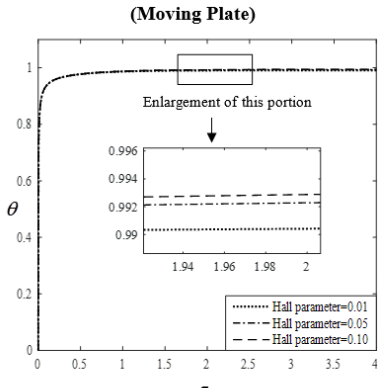

$\tau$

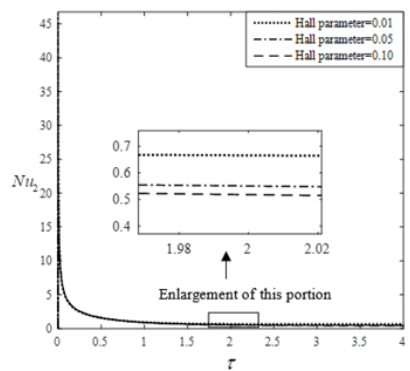

(d)
Figure 11. Effect of Hall parameter $\left(\beta_{e}\right)$ on (a) Temperature (stationary plate); (b) Temperature (moving plate); (c) Local Nusselt number (stationary plate); (d) Local nusselt number (moving plate); where $\mathrm{S}=-1.00, \beta_{i}=3.00, H_{a}=$ 3.00, $R_{e}=2.00, E_{c}=0.01 P_{r}=1.00$ and $\tau_{D}=0.1$

Fig.11 shows that the temperature increases with the increase of Hall parameter $\beta_{e}$ at both stationary and moving plates while the local Nusselt number decreases with the increase of Hall parameter $\beta_{e}$ at both stationary and moving plates.
The temperature and local Nusselt number at moving plate is more than the temperature and local Nusselt number at stationary plate.
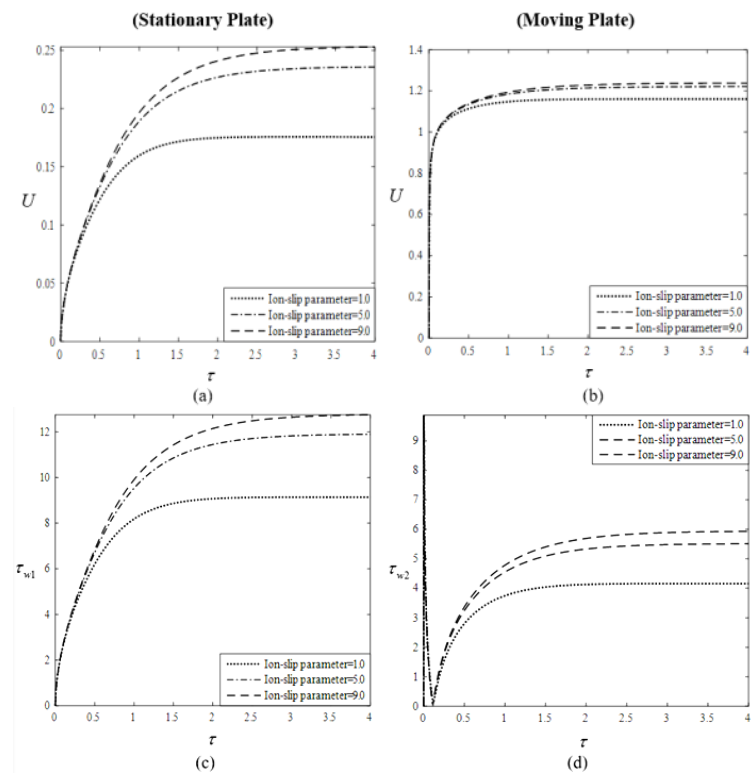

Figure 12. Effect of Ion-slip parameter $\left(\beta_{i}\right)$ on (a) Primary velocity (stationary plate); (b) Primary velocity (moving plate); (c) Local shear stress (stationary plate); (d) Local

shear stress (moving plate); where $S=-1.00, H_{a}=$ 3.00, $\beta_{e}=0.10, R_{e}=2.00, E_{C}=0.01 P_{r}=1.00$ and $\tau_{D}=$ 0.1

Fig. 12 shows that the primary velocity as well as the local shear stress increases with the increase of Ion-slip parameter $\beta_{i}$ at both stationary and moving plates.

The primary velocity at moving plate is more than the primary velocity at stationary plate while the local shear stress at moving plate is fewer than the local shear stress at stationary plate.
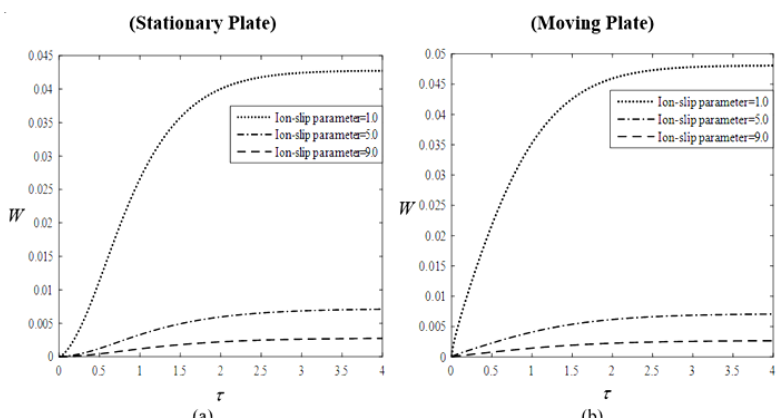

Figure 13. Effect of Ion-slip parameter $\left(\beta_{i}\right)$ on (a) Secondary velocity (stationary plate); (b) Secondary velocity (moving plate); where $\mathrm{S}=-1.00, H_{a}=3.00, \beta_{e}=0.10, R_{e}=$ $2.00, E_{c}=0.01 P_{r}=1.00$ and $\tau_{D}=0.1$

Fig.13 shows that the secondary velocity decreases with the increase of Ion-slip parameter $\beta_{i}$ at both stationary and moving plates.

The secondary velocity at moving plate is more than the secondary velocity at stationary plate. 

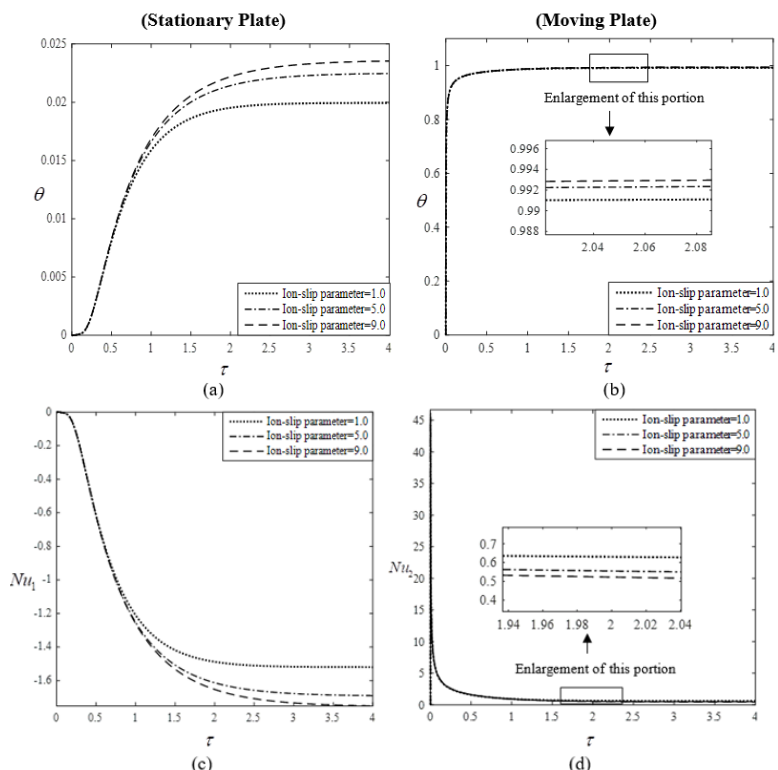

Figure 14. Effect of Ion-slip parameter $\left(\beta_{i}\right)$ on (a)

Temperature (stationary plate); (b) Temperature (moving plate); (c) Local Nusselt number (stationary plate); (d) Local

Nusselt number (moving plate); where $\mathrm{S}=-1.00, H_{a}=$ $3.00, \beta_{e}=0.10, R_{e}=2.00, E_{c}=0.01 P_{r}=1.00$ and $\tau_{D}=$ 0.1

Figure 14 shows that the temperature increases with the increase of Ion-slip parameter $\beta_{i}$ at both stationary and moving plates while the local Nusselt number decreases with the increase of Ion-slip parameter $\beta_{i}$ at both stationary and moving plates.

The temperature and local Nusselt number at moving plate is more than the temperature and local Nusselt number at stationary plate.
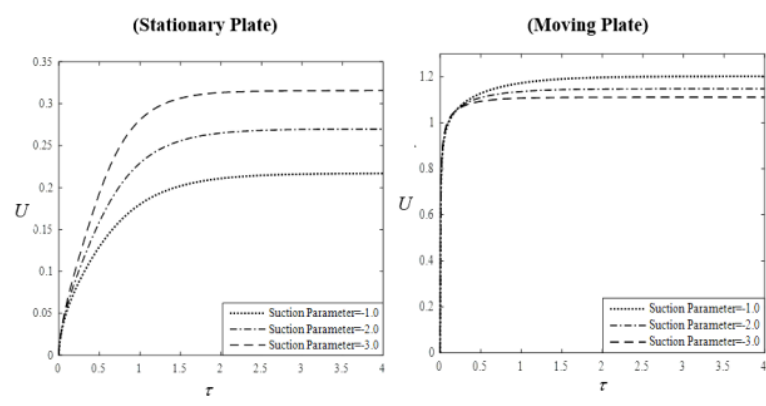

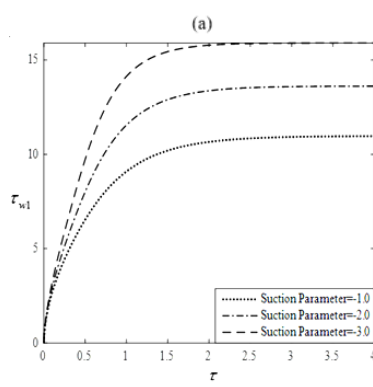

(c)

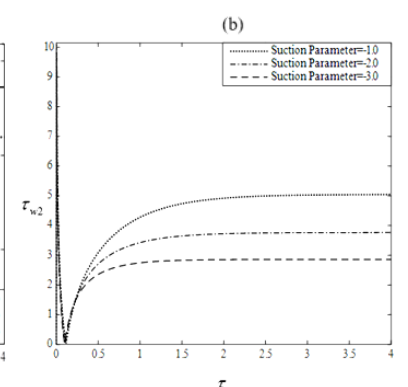

(d)
Figure 15. Effect of Suction parameter (S) on (a) Primary velocity (stationary plate); (b) Primary velocity (moving plate); (c) Local shear stress (stationary plate); (d) Local shear stress (moving plate); where $\beta_{e}=0.10, \beta_{i}=$ $3.00, H_{a}=3.00, R_{e}=2.00, E_{c}=0.01 P_{r}=1.00$ and $\tau_{D}=$ 0.1
Fig. 15 shows that the primary velocity and the local shear stress decreases with the increase of Suction parameter $S$ at stationary plate. Again, the primary velocity and the local shear stress increases with the increase of Suction parameter $S$ at moving plate.

The primary velocity at moving plate is more than the primary velocity at stationary plate while the local shear stress at moving plate is fewer than the local shear stress at stationary plate.
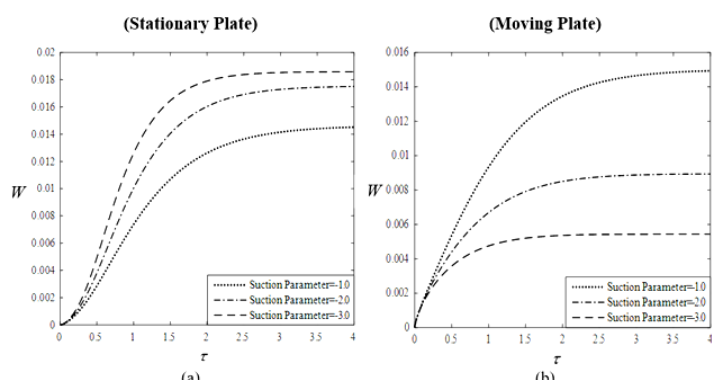

Figure 16. Effect of Suction parameter (S) on (a) Secondary velocity (stationary plate); (b) Secondary velocity (moving plate); where $\beta_{e}=0.10, \beta_{i}=3.00, H_{a}=3.00, R_{e}=$ $2.00, E_{c}=0.01 P_{r}=1.00$ and $\tau_{D}=0.1$

Fig. 16 shows that the secondary velocity decreases with the increase of Suction parameter $S$ at stationary plate while at moving plate it increases with the increase of Suction parameter $S$.

The secondary velocity at moving plate is fewer than the secondary velocity at stationary plate due to Suction parameter $S$.

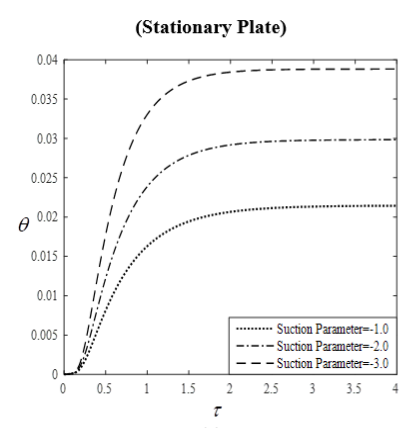

(a)

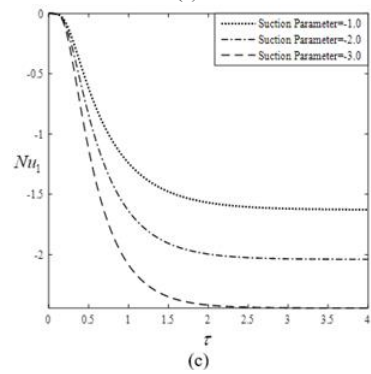

(c)

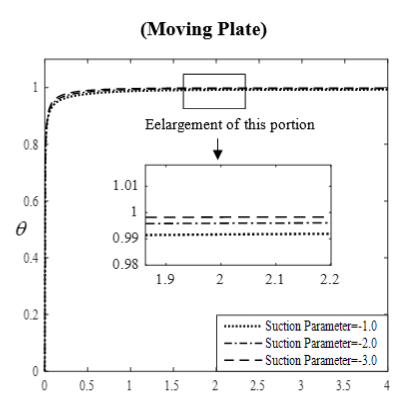

(b)

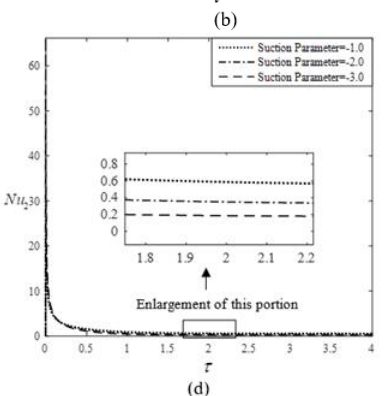

Figure 17. Effect of Suction parameter (S) on (a)

Temperature (stationary plate); (b) Temperature (moving plate); (c) Local Nusselt number (stationary plate); (d) Local

Nusselt number (moving plate); where $\beta_{e}=0.10, \beta_{i}=$ 3.00, $H_{a}=3.00, R_{e}=2.00, E_{c}=0.01 P_{r}=1.00$ and $\tau_{D}=$ 0.1

Figure 17 shows that the temperature decreases with the increase of Suction parameter $S$ at both stationary and moving 
plates while the local Nusselt number increases with the increase of Suction parameter $S$ at both stationary and moving plates.

The temperature and local Nusselt number at moving plate is more than the temperature and local Nusselt number at stationary plate.

\subsection{Comparison}

Finally, qualitative and quantitative comparisons of the steady-state results with the published results of Attia and Sayed-Ahmedm [6] are presented in Figs. 18(a,b) and 19.

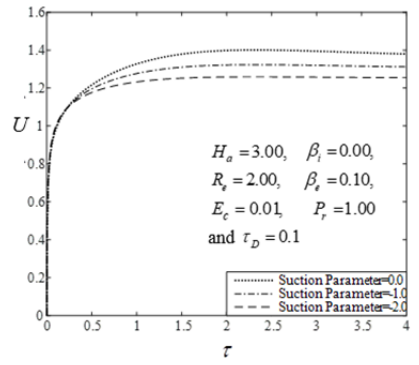

(a) (MATLAB R2015a)

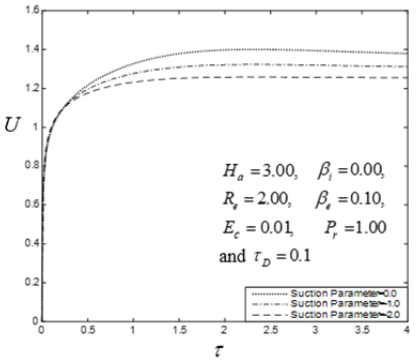

(b) (SDF 6.6a)
Figure 18. Primary Velocity in absence of Ion-slip at moving plate

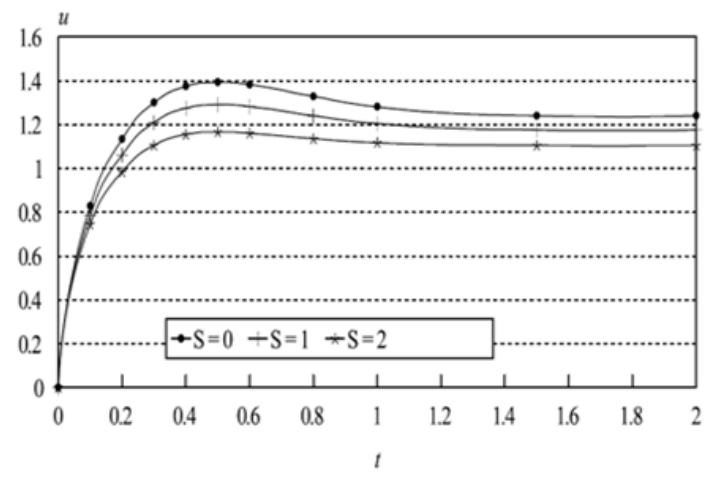

Figure 19. Primary Velocity in absence of Ion-slip at moving plate by Attia and Ahmedm [6]

Thus, the obtained results are qualitatively as well as quantitatively quite same with the results of Attiaand SayedAhmedm [6]. The Crank-Nicolson implicit method has been used for numerical technique by Attiaand Sayed-Ahmedm [6] In the present study the explicit finite difference method has been used for numerical technique and also the MATLAB R2015a and Studio Developer FORTRAN 6.6a (SDF 6.6a) both tools have been used for numerical simulations. Since both tools have shown the same results as shown in Figure 18 $(a, b)$, but the results of Attiaand Sayed-Ahmedm [6] are not exactly quantitatively same. Therefore, the present model simulation tools are the more accurate relating to experimental data.

\section{CONCLUSION}

The explicit finite difference solution technique has been used on unsteady viscous incompressible Bingham fluid flow through parallel plates with Hall current, Ion-slip and suction. The steady state solutions have been obtained. The results were discussed for different values of important parameters as Hall parameter $\beta_{e}$, Ion-slip parameter $\beta_{i}$, where $\alpha_{e}=1+\beta_{i} \beta_{e}$,
Hartmann number $H_{a}$ and Suction parameter $S$. For brevity, the effect of Reynolds number $R_{e}$, Prandtl number $P_{r}$, Eckert number $E_{c}$ and Bingham number $\tau_{D}$ are not shown. The physical properties are investigated graphically for different values of corresponding parameters. Among them some important findings of this investigation are mentioned as follows:

(1). The primary velocity and local shear stress increases with the increase of $\beta_{e}$ and $\beta_{i}$ at both stationary and moving walls while the primary velocity and local shear stress increases with the increase of $S$ at moving wall.

(2). The primary velocity and local shear stress decreases with the increase of $H_{a}$ at both stationary and moving walls while the primary velocity and local shear stress decreases with the increase of $S$ at stationary wall.

(3). The secondary velocity increases with the increase of $H_{a}$ at both stationary and moving walls while at moving wall it increases with the increase of $S$.

(4). The secondary velocity decreases with the increase of $\beta_{e}$ and $\beta_{i}$ at both stationary and moving walls while at stationary wall it decreases with the increase of $S$.

(5). The temperature increases with the increase of $\beta_{e}$ and $\beta_{i}$ while it decreases with the increase of $S$ at both stationary and moving walls.

(6). The temperature decreases with the increase of $H_{a}$ at both stationary and moving walls.

(7). The local Nusselt number decreases with the increase of $\beta_{e}$ and $\beta_{i}$ while it increases with the increase of $S$ at both stationary and moving walls. At moving wall it decreases with the increase of $H_{a}$ while at stationary wall it increases with the increase of $H_{a}$.

(8). The secondary velocity reaches steady state briskly in the comparison with primary velocity and temperature.

\section{ACKNOWLEDGEMENT}

This work is financed and supported by National Science and Technology under Ministry of Science and Technology, Government of the People's Republic of Bangladesh.

\section{REFERENCES}

[1] Bingham EC. (1916). An investigation of the laws of plastic flow. US Bureau of Standards Bulletin 13: 309353.

[2] Buckingham E. (1921). On plastic flow through capillary tubes. ASTM Proceedings 21: 1154-1156.

[3] Bird RB, Dai GC, Yarusso BJ. (1983). The rheology and flow of Viscoplastic materials. Reviews in Chemical Engineering 1(1): 1-70. https://doi.org/10.1515/revce1983-0102

[4] Frigaard IA, Howison SD, Sobey IJ. (1994). One the stability of Poiseuille flow of a Bingham fluid. Journal of Fluid Mechanics 150: 133-263. https://doi.org/10.1017/S0022112094004052

[5] Sablani SS, Shayya WH, Kacimov A. (2003). Explicit calculation of the friction factor in pipeline flow of Bingham plastic fluids: A neural network approach. Chemical Engineering Science 58(1): 99-106. https://doi.org/10.1016/S0009-2509(02)00440-2

[6] Attia HA, Sayed-Ahmedm ME. (2004). Hall effect on unsteady MHD Couette flow and heat of a Bingham fluid 
with suction and injection. Applied Mathematical Modeling 28: 1027-1045. https://doi.org/10.1016/S0307-904X(04)00051-4

[7] Jeng J, Zhu K. (2010). Numerical simulation of Taylor Couette flow of Bingham fluids. Journal of NonNewtonian Fluid Mechanics 165: 1161-1170. https://doi.org/10.1016/j.jnnfm.2010.05.013

[8] Swamee PK, Aggarwal N. (2011). Explicit equations for laminar flow of Bingham plastic fluids. Journal of Petroleum Science and Engineering https://doi.org/10.1016/j.petrol.2011.01.015

[9] Naik SH, Rao KR, Murthy MVR. (2014). The effect of hall current on unsteady MHD free convective Couette flow of a Bingham fluid with thermal radiation. International Journal of Engineering and Advanced Technology 3(6): 1-16. http://citeseerx.ist.psu.edu/viewdoc/download?doi=10.1 .1.676.2276\&rep $=$ rep1\&type $=$ pdf

[10] Parvin A, Dola TA, Alam MM. (2015). Unsteady MHD viscous incompressible Bingham fluid flow with hall current. AMSE Journals -2015-Series: Modelling B 84(1): $\quad 38-48$. http://www.iieta.org/sites/default/files/Journals/MMC/ MMC B/84.1 03.pdf

[11] Sreekala L, Kesavareddy E. (2014). Hall effects on unsteady MHD flow of a Non-Newtonian fluid through a porous medium with uniform suction and injection. IOSR Journal of Mechanical and Civil Engineering (IOSR-JMCE) 11(5): 55-64.

[12] Rees DAS, Bassom AP. (2015). Unsteady thermal boundary layer flows of a Bingham fluid in a porous medium. International Journal of Heat and Mass Transfer 82:

460-467. https://doi.org/10.1016/j.ijheatmasstransfer.2014.10.047

[13] Rees DAS, Bassom AP. (2016). Unsteady thermal boundary layer flows of a Bingham fluid in a porous medium following a sudden change in surface heat flux. International Journal of Heat and Mass Transfer 93: 1100-1106.

[14] Perven R, Alam MM. (2015). Fluid flow through parallel plates in the presence of hall current with inclined magnetic field in a rotating system. AMSE Journals Series: $\quad$ Modelling B $\quad$ 84(1): 49-68. http://iieta.org/sites/default/files/Journals/MMC/MMC B/84.1_04.pdf

[15] Islam MM, Mollah MT, Hasan MS, Alam MM. (2017). Numerical solution of unsteady viscous compressible fluid flow along a porous plate with induced magnetic field. AMSE IIETA Journals-2017-Series: Modelling B 86(4): 850-863. 